\title{
Adenomyoepithelioma of breast: case report
}

\author{
K. Sivasuganthan, S. Raviraj \\ Professorial surgical unit, Teaching hospital Jaffna. Sri Lanka
}

Keywords: Breast lump; adenomyoepithelioma; AME; myopeithelial proliferation; immunohistochemistry

\section{Introduction}

Adenomyoepithelioma (AME) is a biphasic tumour of breast composed of variable number of myoepithelial cells around small epithelial lined spaces. Breast acini and ducts usually contain epithelial cells internally which are covered by myoepithelial cells externally. Breast AME typically presents as single unilateral painless lump which usually located in a peripheral portion of the breast [5]. It has characteristic dual proliferation of glandular and myoepithelial cells and has been described by Hamperl [1], in 1970.

Benign myoepithelioma neoplasm, hyperplasia and malignant neoplasm with myoepithelial differentiation are the different types of AME. These neoplasms usually show squamous, chondromyxoid, plasmacytoid, clear cell and myoid spindle cell differentiation of myoepithelial cells. Papillary architecture is seen in most tumours, therefore it's also considered to be a variant of intraductal papilloma [2].

Most of the reported cases were female except for few male patients [3]. Although it is a benign neoplasm, failure to achieve an adequate resection margin may lead to recurrence [4]. Therefore, local resection with free margins is the treatment of choice for these patients. Malignant transformation rarely occurs $[2,4]$.

\section{Case report}

We describe a 31 year-old Sri Lankan Tamil female patient who is generally good health with no family history of cancer. She presented with a left side breast lump for one month duration which she noticed while having a bath. She underwent clinical examination and found to be having two breast lumps at 9'o clock and 2'o clock position without any axillary lymphadenopathy on either side.

Correspondence: .K Sivasuganthan

E-mail: suthan367@gmail.com

Received: 10-06-2017 Accepted: 02-07-2017

(iD http://orcid.org/0000-0002-2748-0767

DOI: http://doi.org/10.4038/sljs.v35i2.8388

The Sri Lanka Journal of Surgery 2017; 35(2): 26-27
Ultrasonographic examination revealed two well defined lesions seen in left breast at 9'o clock and 2'o clock position which favoured fibroadenomata. Her abdominal ultrasound revealed multiple fibroids in the uterus. She underwent a fine needle aspiration of the lesion which revealed blood and fibro-fatty fragments only (C1). Core biopsy was performed which showed few breast acini in a fibro fatty stroma with no evidence of insitu or invasive malignancy. It was believed that the target lesion had been missed and advised on a repeat biopsy. However the patient did not consent for repeated ultrasound guided biopsy.

So we performed excision biopsies of both lumps. They were macroscopically irregular, firm, pale brown tissue masses measuring $55 \times 45 \times 45 \mathrm{~mm}, 35 \times 40 \times 30 \mathrm{~mm}$ and cut sections revealed a well-defined heterogeneous lesion. Microscopically there was circumscribed lesion with lobules of small and intermediate size ducts with prominent myoepithelial cells lining without cellular atypia. Background mammary tissue showed features of sclerosing adenosis and fibrocystic disease and concluded as adenomyoepithelioma. Patient was reassured about her condition and followed up in our surgical clinic three monthly for routine examination of her breast to identify local recurrence. Also she was educated on self-breast examination.

\section{Discussion}

Myoepitheliosis, adenomyoepithelioma, and myoepithelial carcinoma are various types of myoepithelial tumours. AME is characterized by proliferation of an inner epithelial cellular layer with peripherally situated myoepithelial cellular layer. Most of the tumours are benign, sporadic and rarely can it be malignant. The aetiology of the lesion is still unknown. Reported cases shows that majority of affected are in the fifth to sixth decade of life [6].

Clinical and radiological observation is inadequate for diagnosis, therefore histology needs to be obtained to confirm the diagnosis. Mammographically, it may show probably benign features (BIRADS III) while others have features suspicious for malignancy (BIRADS IV).

Adenomyoepithelioma should be differentiated from tubular adenoma, fibrodenoma pleomorphic adenoma and sclerosing 
adenosis of breast [6].

Epithelial differentiation can be identified by immunostaining of smooth muscle actin and calponin. proteins. There are various markers used to identify myoepithelial cells, S100 protein is one of them and others are P63, smooth muscle actin, calponin, 34ßE12, CK5/6 [7], but these immuneactivities also observed in epithelial cells as well. Hence these markers are not reliable to identify myoepithelial cells. Recognition of biphasic cellular proliferation in histology with supportive immunohistochemistry aids diagnostic of AME. Immunohistochemistry is not freely available and selected patients undergo this investigation in our institution only for confirmation.

High mitotic rate, cytological atypia and peripheral infiltration are the potential malignant predictors in AME. Therefore, pathology report should be carefully studied to identify atypical features. To avoid recurrence and metastasis, lesion should be completely excised with margins. The prognosis of this condition is usually good.

Our patient had well defined lesions, which were excised with adequate margin, so risk of recurrence is relatively small. We are following up in surgical clinic to make sure that there is no recurrence. Also the patient is educated on self-breast examination for early detection of recurrence.

\section{Conclusion}

Adenomyoepithelioma is a benign breast lesion which can recur locally and transform into malignancy with or without metastasis. Combination of histology and immunohistochemistry is important in diagnosis. In case of failed biopsy, ultrasound guided biopsy should be performed for accurate diagnosis. Complete excision of tumour with margin is important to prevent recurrence. Self-breast examination may have a role in early detection of tumour recurrence.

\section{References}

1. Hamper H, The myoepithelia (myoepithelial cells): normal state; regressive changes; hyperplasia; tumours. Curr Top Pathol, $1970 ; 53: 161-213$

2. Rosen PP, Adenomyoepithelioma of the breast. Human pathology. 1987; 18(12):1232-7.

3. Tamura G, Monma N, Suzuki Y, Satodate R, Abe H: Adenomyoepithelioma (myoepithelioma) of the breast in a male. Hum Pathol 1993; 24:678-681.

4. Loose JH, Patchefsky AS, Hollander IJ, Lavin LS, Cooper HS, Katz SM: Adenomyoepithelioma of the breast. A spectrum of biologic behaviour. Am J Surg Pathol 1992; 16:868-876.

5. Hoda SA, Rosen PP. Observations on the pathologic diagnosis of selected unusual lesions in needle core biopsies of breast. Breast J. 2004; 10(6):522-527.

6. Milind A Bhatkule, Manjusha S Dhawle, Rajan S Bindu, Adenomyoepithelioma of breast: Report of a rare case, clinical cancer investigation journal, 2013; Vol2(1) : 57-59

7. Jian Zhu, Gaofeng Ni, Dan Wang, Qingqing He, Peifeng Li, Lobulated adenomyoepithelioma: a case report showing immunohistochemical profiles, Int J Clin Exp Pathol 2015;8(11):15407-15411

\section{Key Points:}

- Adenomyoepithelioma is a benign tumour with malignant potential

- Histology and immunohistochemistry is important in diagnosis

- Complete excision with margins is mandatory to prevent recurrence 